\title{
Stratification of Cardiovascular Diseases Using Deep Learning
}

\author{
Bhanu Prakash Doppala $^{1 *}$, Debnath Bhattacharyya ${ }^{2}$, Midhun Chakkravarthy $^{1}$ \\ ${ }^{1}$ Department of Computer Science and Multimedia, Lincoln University College, Kuala Lumpur 47301, Malaysia \\ ${ }^{2}$ Department of Computer Science and Engineering, K L Deemed to be University, KLEF, Guntur 522502, India
}

Corresponding Author Email: bhanu.doppala@lincoln.edu.my

https://doi.org/10.18280/ria.340401

Received: 15 June 2020

Accepted: 10 August 2020

\section{Keywords:}

Cardiovascular Diseases (CVD), deep

learning, cat fuzzy neural network, hybrid ant colony, African buffalo optimization

\begin{abstract}
Heart-based diseases are one of the causes for major death rate in the world. WHO (World Health Organization) specified that 17 million of people are losing their lives per year due to several heart diseases. Artificial Intelligence playing a prominent role in disease identification and prediction from medical data. Magnetic Resonance Imaging plays a vital role in producing detailed images of internal organs and soft tissues for better understanding the condition. Magnetic Resonance Image contains more noisy data this is one of the issues to be addressed, hence this research focuses on the prediction of cardiovascular diseases using an innovative hybrid algorithm and addresses the issue of noise using Hann filters. A Hybrid algorithm is proposed with combination of Cat Fuzzy Neural Model (CFuNM) and Hybrid Ant Colony and African Buffalo Optimization. Cat Fuzzy Neural Model (CFuNM) is used to classify cardiac diseases such as cardiomyopathy, pericardial effusion, coronary artery, amyloidosis, and other coronary heart diseases and for the severity analysis of disease we used Hybrid Ant Colony and African Buffalo Optimization (HAC-ABO) mechanism. This research of Hybrid deep learning model improved the classification accuracy of $99.3 \%$ and error rate of $0.18 \%$ which is considerably good when compared to existing methods.
\end{abstract}

\section{INTRODUCTION}

Artificial Intelligence refers to several applications of machines that conduct activities that are characteristic of individual intellect, e.g. presume final thoughts coming from deduction or even logical reasoning, while Machine Learning is actually an extra limited kind of computational mechanism which makes use of an algebraic design along with instruction information to discover exactly how to produce prophecies. As opposed to clearly calculating arise from a collection of predefined regulations, Machine Learning knows criteria coming from instances as well as consequently possesses the possibility to conduct much better at a job like identifying as well as classifying information through being actually left open to an extra instance. One of the most innovative Machine Learning methods, likewise referred to as Deep learning (DL), are actually appropriated for this reason which has been clearly indicated in Figure 1.

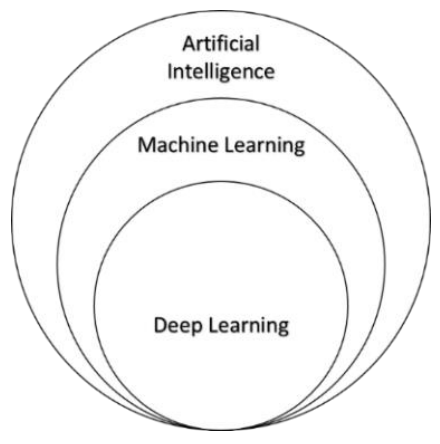

Figure 1. Evolution of deep learning
Deep learning models are actually one encouraging opportunity of research study in to the automated removal of sophisticated information portrayals (attributes) at extreme degrees of absorption. Such protocols create a split, ordered design of knowing as well as embodying information, where higher-level components are actually determined in regards to lower-level components. The ordered understanding style of Deep Learning protocols is actually inspired through expert system mimicing deep layered learning, split understanding procedure of the main sensorial locations of the neocortex in the individual mind, which instantly draws out components as well as absorptions coming from the rooting records Deep Learning methods are actually pretty useful when handling large amount of unsupervised data, and also commonly discover records depictions in a greedy layer-wise manner [1].

In medical industry early identification of a disease became challenging task. Making prediction or conclusion based on the available data became too difficult. In this regard we need a decision making system which can help health experts to identify any disease more accurately. One of such dangerous diseases is Heart based diseases. Several Millions of people losing their lives because of Heart based diseases. This may be caused because of their daily life style or may be because of prevailing situations. Lot of research has been done on this area where several prediction models has been generated on different available datasets.

In certain for this research study MRI images are utilized due to the fact that an MRI is extremely experienced at recording photos that aid medical professionals figure out if there are uncommon cells within the body system. MRIs are actually even more outlined in their photos. If you require an 
additional in-depth picture of your smooth cells, tendons, or even body organs, your medical professional is going to typically propose an MRI.

\subsection{Hann Filter}

Hanning Filter is relatively simple low pass filter that can be described by single parameter, The Hanning Filter is defined in the frequency domain as follows:

$$
H(f)=0.54+0.46 \cos (\text { pi.f } / \mathrm{fm}) 0<=|\mathrm{f}|<=\text { fm otherwise }
$$

where, $f$ is the spatial frequencies of the image and fm the cutoff (critical) frequency. In signal processing, the Hann window is a window function, called the Hann function, named after Julius Ferdinand von Hann, an Austrian meteorologist. The usage of the Hann window is called "Hanning", as a signal to apply the Hann window to a digital signal processing [2]. The Hanning (Hann) Filter is very effective in reducing image noise as it reaches zero very quickly; however, it does not preserve edges [3, 4].

\subsection{Hybrid optimization algorithms}

Combination protocols are actually 2 or even more protocols that fuse as well as suit one another to make a lucrative unity coming from their assimilation [5]. Which are generally well known as hybrid metaheuristics (HMs). It is well known for its performance in handling noise, uncertainty, vagueness, and imprecision $[6,7]$. For the work carried out here, instead of using HM, we prefer to use the general term hybrid algorithms to refer to the similar notion [8]. The hybrid algorithm named ACO-ABO combines the framework of Ant Colony Optimization (ACO), African Buffalo Optimization (ABO) algorithms to find the optimized solution for the system. The ACO algorithm is used to find the initial solution set, the $\mathrm{ABC}$ algorithm is employed to test and improve each of the probable solutions provided by the ABO module [9].

Medical image processing becomes familiar in the current decade thus medical imaging is the method for generating the visual images of an inside body part for medical investigation and medicinal interference. In addition, the medical imaging process contains disease classification and managing also it is utilized to gather the information of the inner organs [10], which involves organic and radiological imaging it can be used as electromagnetic, thermal, magnetic and isotope imaging [11]. Many methods are used for the confirmation of information available about the function of the body [12]. Every year, many images are generated globally for various usages of diagnostics. This image process will be developed the image of the interior arrangement of the body without enveloping actions. Medical imaging should be developed quickly due to an increased number of image processing techniques like image analysis, image recognition and image enhancement. This image processing used to increase the fraction and quantity of detected images. The medicinal image processing handling images and it involves some operations for example image gaining, storage, communication and presentation. In addition, the purpose of digital images can be used for many benefits like cheap processing cost, quicker, immediate quality assessment, cheap reproduction, and adaptable manipulation.

Medical image processing is a significant role to predict and identify Cardiovascular Diseases (CVD). CVD is the most significant disease [13]; a statistical report says that approximately $31 \%$ of deaths happening around the world per year because of CVD. These CVD are identified using the tests like electrocardiogram (ECG), cardiac Magnetic Resonance Imaging (CMRI), etc. that can detect the heart diseases and abnormal heartbeats. Also, CMRI evaluates the structural and hemodynamic features of the human heart. Process of the MRI and disease prognosis is represented in Figure 2.

Moreover, this process is done over MRI heart images and this information defeated the challenges in cardiac imaging and several imaging modalities are echocardiography and fluoroscopy in interventional procedures. So, the hybrid deep learning modules can enhance the classification accuracy and prediction rate. Hence, this approach introduces the innovative deep learning model for predicting and classifying the cardiovascular diseases.

The classification of cardiac diseases is generally based on cardiac visualization, coronary artery inspection and the computation of ejection fraction for the ventricles. Generally, CVD involves Ischemic Heart Disease (IHD), stroke and Congestive Heart Failure (CHF). Also, are classified based on the heartbeat rate and the heartbeat rate of the ordinary person is between $60-100$ beats per minute.

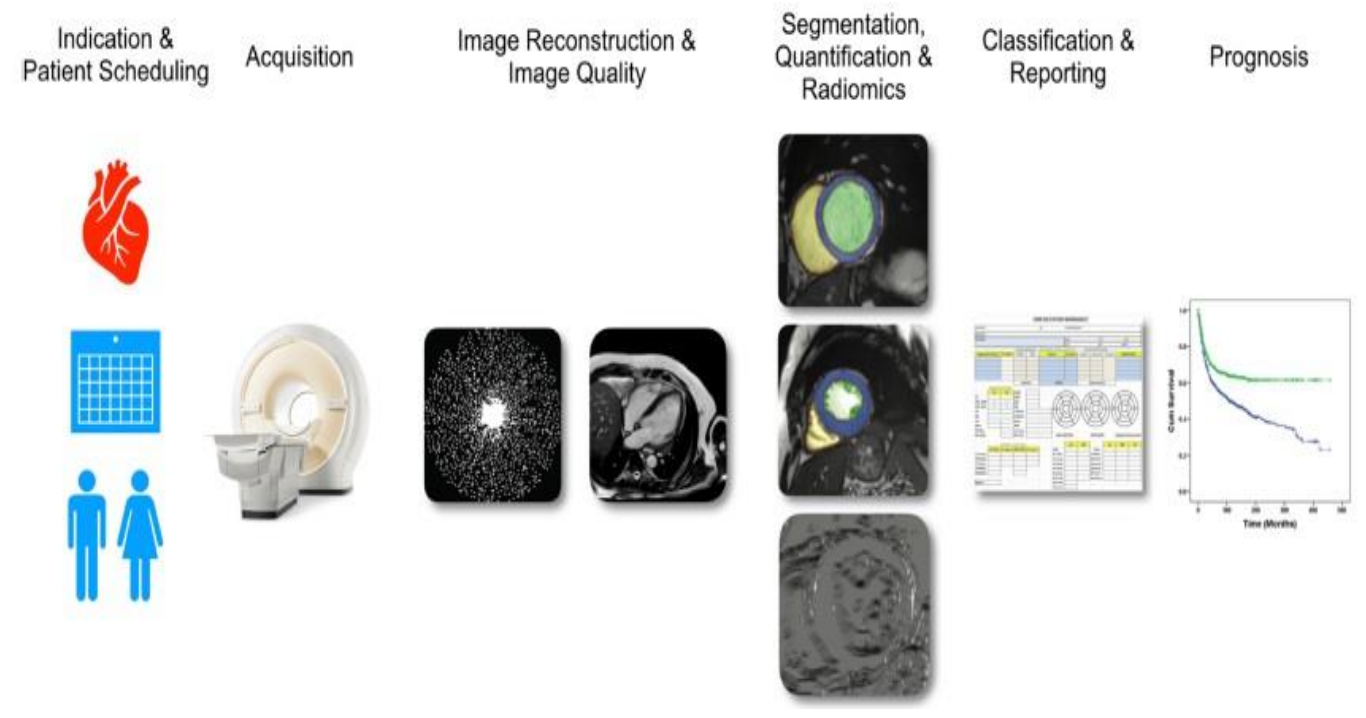

Figure 2. Magnetic resonance imaging as a result of image analysis and also diagnosis 


\section{RELATED WORK}

Cardiovascular (CV) diseases are predicted using the medical images and the method of deep learning can be used for the classification of medical images. The medical imaging and deep learning algorithms are the most useful factors for the prediction of cardiovascular imaging. It creates an impact on developing the factors so Litjens et al. [14] explained the method for state-of-the-art deep learning in cardiovascular imaging. This will explain the categorization of the cardiovascular images and identifying the aspects of the diseases.

The classification and prediction of CVD and other heartbased diseases using deep learning and other conventional technique is a difficult task in medical image processing. For this reason, Khanna et al. [15] proposed the B-mode US technique to improve the medical images and the $\mathrm{CV}$ risk stratification mechanism, thus the efficiency of developed modules is compared with conventional $\mathrm{CV}$ danger estimations.

The classification of $\mathrm{CV}$ diseases from the ECG signal is crucial thus quick corrective treatment of patients is required. So, Hasan et al. [16] presented the technique for categorizing the heart diseases using dimensional deep CNN and the input signal can be passed to the network using a modified ECG signal. This approach achieved the accuracy values as $97.70 \%$, 98.71\% and $98.24 \%$ for MIT-BIH, St-Petersburg, and PTB database.

The fragmentation of the left ventricle from the Cardiac MRI pictures is a step for the calculation of medical indices like ejection division and stroke quantity. Hence, Luo et al. [17] introduced the method that combined with SV segmentation and Hierarchical Extreme Learning Machine (HELM). It increases the accuracy of integrating the LV location method.

Podder et al. [18] narrated their work towards the comparative analysis on Hamming, Hanning and Blackman window. Which helped us to identify that our work can be implemented on Hanning window for fulfilling our requirement.

In this specific paper authors proposed a CNN which will be actually utilized for deep characteristic abstraction as well as LSTM is made use of for decision making from the abstracted characteristics. The speculative outcomes present that their work accomplished a reliability of $99.4 \%$, AUC of $99.9 \%$, uniqueness of $99.2 \%$, level of sensitivity of $99.3 \%$, as well as F1-score of $98.9 \%$ [19].

The efficiency of several computational techniques is actually reviewed making use of Cleveland heart problem dataset which are actually fetched from the UCI machine learning repository along with numerous assessment approaches. Coming from the research, it can be carried out that the best precision of $98.15 \%$ secured through deeper semantic network along with sensitiveness as well as preciseness $98.67 \%$ as well as $98.01 \%$ specificity [20].

\section{PROPOSED METHODOLOGY (CFuNM with HAC- ABO)}

The prediction of cardiovascular diseases contains high difficulties because MRI images have more noise. So, the proposed approach overcomes the difficulties and classifies CVD. Initially, Heart-based MRI images are trained to the system and the unwanted noises are removed by innovative hann filter. Consequently, the deep learning approach as CFuNM [21] is processed over the MRI dataset to classify the disease types such as heart attack, stroke, arrhythmias, etc. In addition to this, severity analysis of disease is done using Hybrid Ant Colony and African Buffalo Optimization (HACABO) mechanism [22]. Moreover, the optimization mechanism segments the affected part also enhances the classification accuracy rate, the proposed methodology is shown in Figure 3.

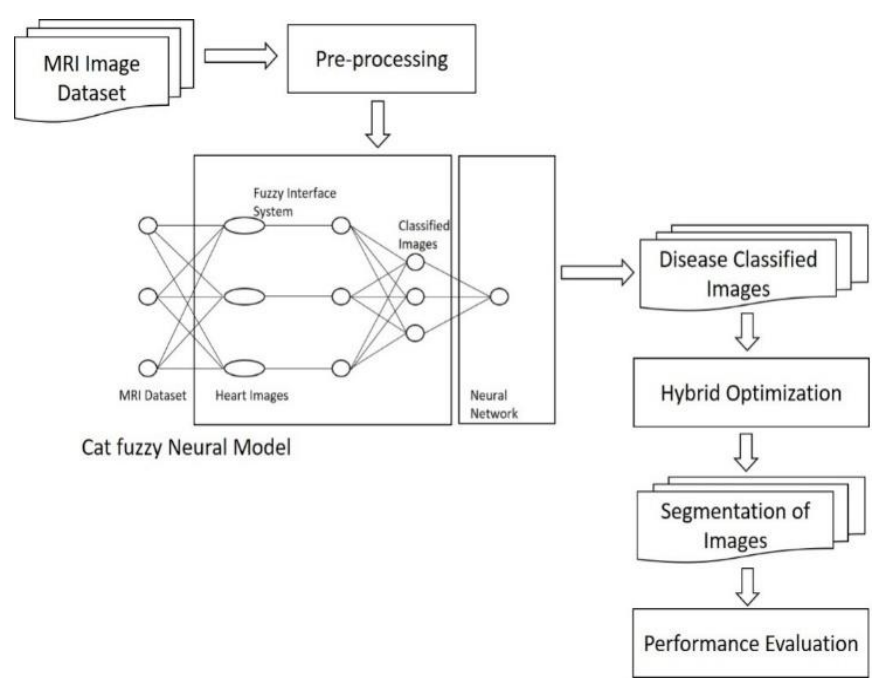

Figure 3. Proposed CFuNM with HAC-ABO

\subsection{Cardiac Magnetic Resonance Imaging (CMRI)}

The present work carried out on a dataset provided by York University Cardio Vascular Dataset for MRI images with 20 frames and 8-15 slices total 32 subjects [23]. Normally the MRI datasets are collected from a net source which contains more noises so pre-processing is needed. Moreover, cardiac image processing is applied for detecting, classification, and diagnosis of heart disease. CMRI can identify and monitor the heart diseases and estimate the heart structure and that produces the most excellent images of the heart for definite circumstance and not used ionizing radiation. CMRI can evaluate the anatomy and utility of the heart chambers, heart valves, size of blood flow through main vessels and the surrounding structures like pericardium. ECG gating and elevated chronological declaration protocols are used for converting the MRI series for modified cardiac imaging.

\subsection{Pre-processing}

MRI images are affected by irrelevant and unpredictable noisy data for example speckle sound and Gaussian noise that are reducing the diagnosis value of the images. Speckle noise highly affected the contrast resolution of the MRI images. So, the innovated Hann filter is used for noise reduction in MRI heart images. Also, Hann filter is used to reduce the sound in a picture and decreases the spatial intensity derivatives present in the image. This filter is used to replace every pixel value into a mean value of image with its neighbours and that neglects the pixel value which is unreliable of their backgrounds of images. The exertion of the temperature of the device inhomogeneity in a magnetic field, imperfect scanner and the movement of patients in the scanning process creates the noises in the MRI images. Image resolution and noiseless 
methods used for the achievement of the high-quality MRI images.

\subsection{Cat Fuzzy Neural Model (CFuNM)}

CFuNM algorithm is one of the gradient approaches; the main purpose of using CFuNM in neural architecture is to reduce training error also makes the classification process easier. Moreover, the presence of CFuNM in deep learning approach can attain improved classification accuracy and precision rate. CFuNM is the dynamic structural design that is combined for reduce in size and develop the rule nodes. The created rule nodes and link added without difficulties and not including with interrupted existing nodes. These new nodes are generated through learning which is signifying the fuzzy label neurons that can be personalized through learning. Neurons used to represent the fuzzy morals of the variable and attached with Membership Functions (MF).

\subsection{Segmentation}

Segmentation is mainly used for the segment of the affected part of the diseases from the MRI image. In this proposed approach, image segmentation is done with the use of innovative HAC-ABO. Segmentation is the progression of changing the illustration of an image for easy to analyse. Also, image segmentation is mainly used for situate objects and limitations similar to lines and curves in the images. It separates the heart images into numerous divisions or set of pixels and also allocates the label to pixels in images. Some of the pixels have a similar label assign definite features.

\section{Algorithm 1: HAC-ABO for CVD segmentation}

Step 1: Identifying the CVD affected part location from MRI image.

Step 2: Finding the accurate position of the disease.

Step 3: Classifying the Cardiovascular Disease.

Step 4: Segment the affected part if yes proceed to next step, other wise jump to step 3 .

Step 5: Removing the noise from the MRI images.

Step 6: Generating the classified image. $(0 / 1)$

The classification of CVD is necessary for medical imaging because millions of peoples are affected by heart diseases. Thus, the proposed CFuNM with HAC-ABO approach classified the CVD and segmented the affected part from the MRI image. The implementation results provide the effectiveness of the proposed CFuNM with HAC-ABO method.

\section{RESULTS AND DISCUSSION}

The Proposed method is validated on a dataset provided by York University Cardio Vascular Dataset for MRI images with 20 frames and 8-15 slices total 32 subjects. The proposed model is implemented using MATLAB R2020a version in windows 10 environment $2.60 \mathrm{GHz}$ Intel Core-i7 processor with $8 \mathrm{~GB}$ RAM. The proposed CFuNM with HAC-ABO algorithm is utilized to classify and quantify the severity of cardiovascular diseases.

The implementation results are discussed as follows, and the original input MRI heart image is obtained from various patients are shown in Figure 4. Also, these input MRI images contain more noise. So, these images are filtered using the Hann filter.

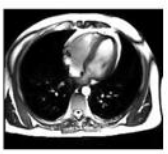

( a )

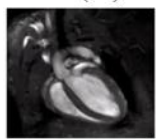

(f)

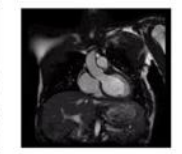

(b)

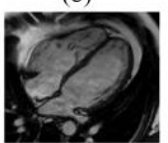

(g)

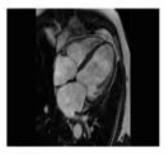

(c)

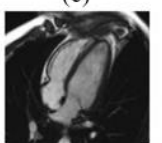

(h)

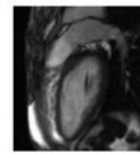

(d)

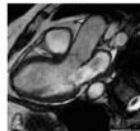

(i)

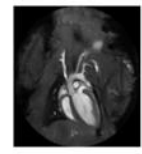

(e)

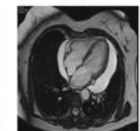

(j)
Figure 4. (a). MR Image of heart for patient A, (b). MR Image of heart for Patient B, (c). Input MR Image of heart for patient $C,(d)$. MR Image of heart for Patient D, (e). MR Image of heart for Patient E, (f). MR Image of heart for Patient F, (g). MR Image of heart for Patient G, (h). MR Image of heart for Patient H, (i). MR Image of heart for Patient I, (j). MR Image of heart for Patient J

\subsection{Pre-processing}

Pre-processing is the method of removing the unwanted noise in an image. The dataset contains patient records with MRI that contains more noise because of the moving behavior of the patients. Also, pre-processing is necessary for noisy data inconsistent, and incomplete data. Generally, the MRI images contain artefacts from patient and equipment-based noises, volume effect artefacts. To avoid these artefacts, the proposed approach introduced the novel Hann filter.

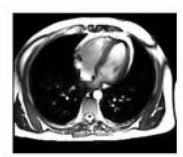

( a )

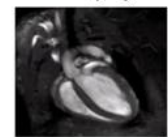

(f)

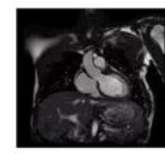

(b)

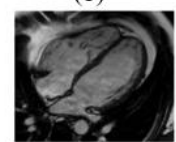

(g)

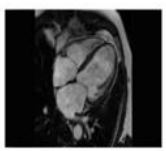

(c)

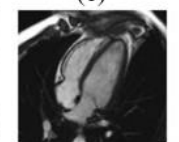

(h)

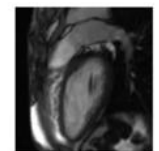

(d)

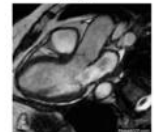

(i)

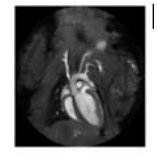

(e)

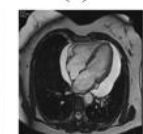

(j)
Figure 5. (a). Filtered MRI Image of heart for patient A, (b). Filtered MRI Image of heart for patient B, (c). Filtered MR Image of heart for patient C, (d). Filtered MRI Image of heart for patient D, (e). Filtered MRI Image of heart for patient $\mathrm{E}$,

(f). Filtered MRI Image of heart for patient F, (g). Filtered

MRI Image of heart for patient G, (h). Filtered MRI Image of heart for patient $\mathrm{H}$, (i). Filtered MRI Image of heart for patient I, (j). Filtered MRI Image of heart for patient J

Also, this hann filter is used for removing unwanted noises in the MRI dataset. Also, it reduced the distortions in the images finally it provides the noiseless clear images. Moreover, the pre-processed MRI image for various CVD patients is shown in Figure 5.

\subsection{Classification}

Classification of CVD is very necessary for medical imaging for identifying the disease's type and illness of the patients. Many classifiers are used for disease classification and CVD classification. In this approach, introduced a new 
deep learning approach as the CFuNM model for classifying the diseases. The MRI images are processed in the deep learning model thereafter that creates clear disease classified images. Also, it was identifying the heartbeat rate of CVD patients for categorizing cardiovascular diseases.

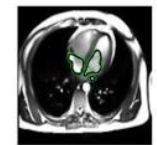

(a)

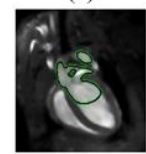

(f)

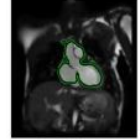

(b)

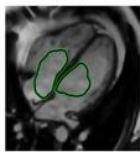

(g)

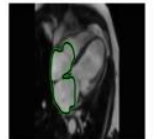

(c)

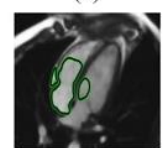

(h)

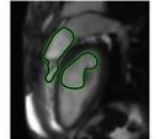

(d)

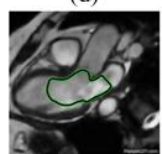

(i)

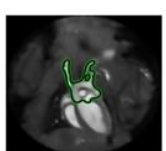

(e)

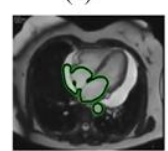

(j)
Figure 6. (a). Classified MRI Image of heart for patient A, (b). Classified MRI Image of heart for patient B, (c).

Classified MRI Image of heart for patient C, (d). Classified MRI Image of heart for patient D, (e). Classified MRI Image of heart for patient E, (f). Classified MRI Image of heart for patient F, (g). Classified MRI Image of heart for patient G,

(h). Classified MRI Image of heart for patient H, (i).

Classified MRI Image of heart for patient I, (j). Classified MRI Image of heart for patient $\mathrm{J}$

Moreover, CFuNM improves the classification accuracy and precision rates. The CVD are classified for various patients that are shown in Figure 6.

\subsection{Segmentation}

Image segmentation is necessary process in medical image processing that is used for observe information about the diseases. CVD are classified using CFuNM approach and finally, the affected parts are segmented using the hybrid Ant colony African buffalo Optimization technique that is shown in Figure 7.

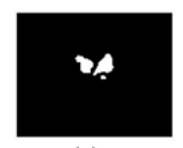

(a)

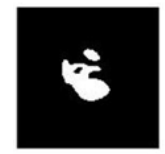

(f)

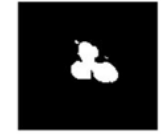

(b)

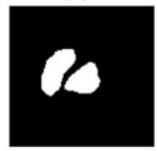

(g)

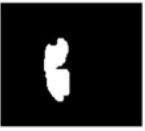

(c)

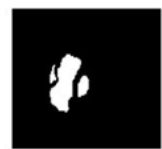

(h)

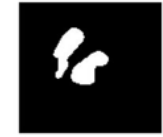

(d)

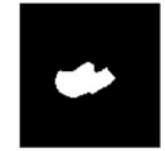

(i)

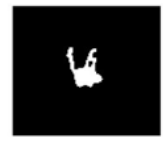

(e)

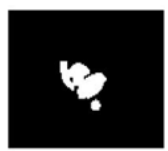

(j)
Figure 7. (a). Segmented affected part image for patient A, (b). Segmented affected part image for patient B, (c). Segmented affected part image for patient C, (d). Segmented affected part image for patient D, (e). Segmented affected part image for patient E, (f). Segmented affected part image for patient F, (g). Segmented affected part image for patient

G, (h). Segmented affected part image for patient H, (i).

Segmented affected part image for patient I, (j). Segmented affected part image for patient $\mathrm{J}$

The effectiveness of the planned model is evaluated by recent existing works in terms of Accuracy, Precision, Specificity, and Sensitivity. The accuracy can be calculated using Eq. (1)

$$
A C C=\frac{T N^{\prime}+T P^{\prime}}{T N^{\prime}+T P^{\prime}+F N^{\prime}+F P^{\prime}}
$$

Here is true negative, stand for true positive, denote the false negative and stand for false positive

Precision is used for measuring the effectiveness of the disease classification then the ability of the classifier to classify the disease calculation is done by the recall, which is measured using

$$
\begin{gathered}
\text { precision }=\frac{T P^{\prime}}{T P^{\prime}+F P^{\prime}} \\
\operatorname{Re} \text { call }=\frac{T P^{\prime}}{T P^{\prime}+F N^{\prime}}
\end{gathered}
$$

Sensitivity has the ability to identify those with diseases. Specificity has the ability to identify those without diseases. The sensitivity and specificity can be calculated using

$$
\begin{aligned}
& S E N=\frac{T P^{\prime}}{T P^{\prime}+T N^{\prime}} \\
& S P E C=\frac{T N^{\prime}}{F P^{\prime}+T N^{\prime}}
\end{aligned}
$$

Mean absolute error has defined as the identification of absolute variation between the actual recommendations done by a cardiologist and recommendation prepared by a recommender system. Also, imaging plays a crucial role in a diagnostic manner for many patients. Also, the evaluation of normal diagnostic error rates is ranging from $3 \%$ to $5 \%$. The error rate can be computed as,

$$
\text { Error_rate }=\frac{F P^{\prime}+F N^{\prime}}{\text { Total }}
$$

This method provides the better results for prediction of different CVD. Various diseases are predicted depending on the heartbeat rate. Prediction of some CVD like WPW syndrome, Bradycardia, angina and Coronary Artery diseases provides better performance compared to other methods.

Wolff-Parkinson-White syndrome is a condition characterized by abnormal electrical pathways in the heart that cause a disruption of the heart's normal rhythm. The performance results for predicting WPC syndrome is exposed in Figure 8.

In this approach, the classification of WPW syndrome provides $99.83 \%$ accuracy, $99.98 \%$ specificity, $99.11 \%$ sensitivity, $81.8 \%$ precision rate, $85.99 \%$ recall and $0.188 \%$ lower error rate that results are mentioned in Table 1. Performance analysis of model has been represented using ROC curves (Figure 9).

Bradycardia is a heart rate that's too slow. In general, a resting heart rate of fewer than 80 beats per minute (BPM) qualifies as bradycardia. The prediction results of Bradycardia are mentioned in Figure 10. 
Table 1. Performance results for WPW syndrome

\begin{tabular}{cc}
\hline Performance Metrics & Percentage \\
\hline Accuracy & 99.83 \\
Specificity & 99.98 \\
Sensitivity & 99.11 \\
Precision & 81.8 \\
Recall & 85.99 \\
Error rate & 0.188 \\
\hline
\end{tabular}

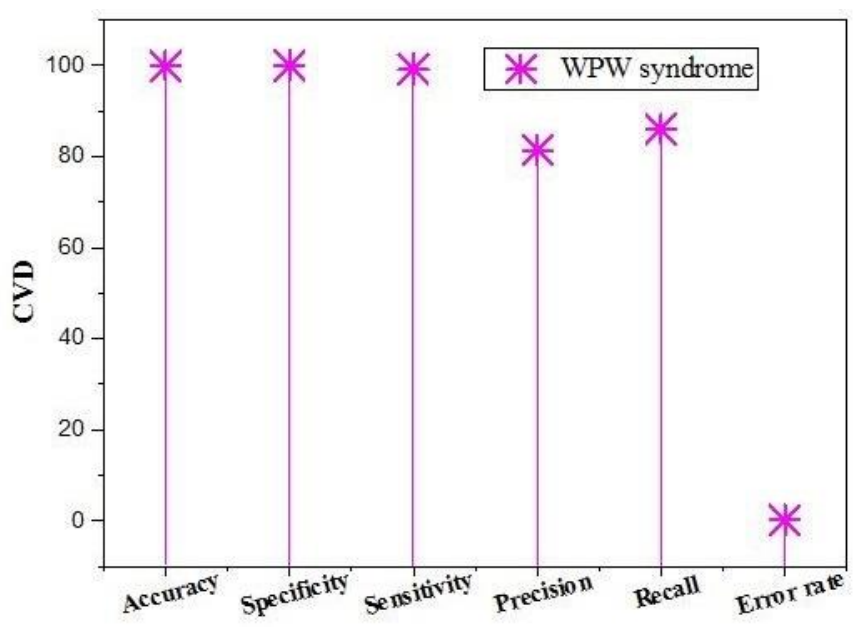

Figure 8. Performance results for predicting WPC syndrome

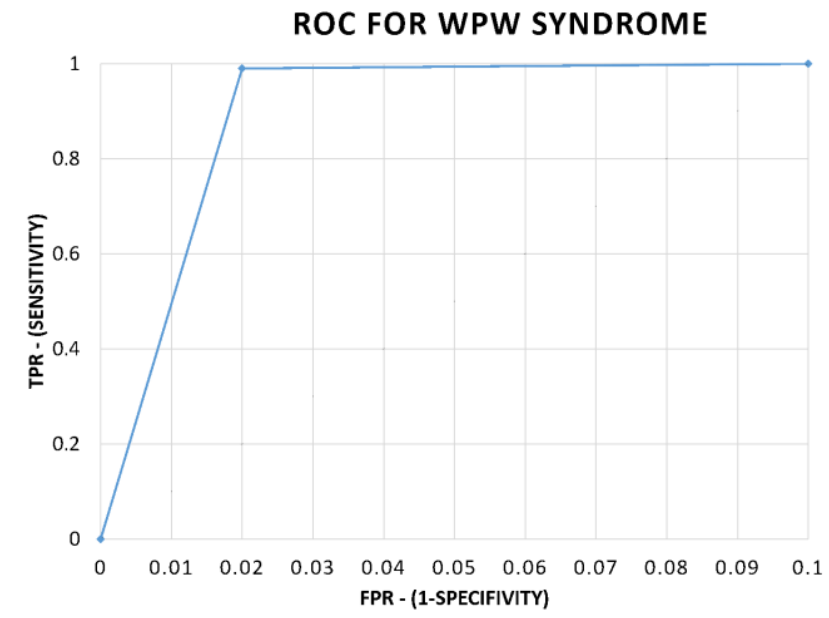

Figure 9. ROC for WPW syndrome

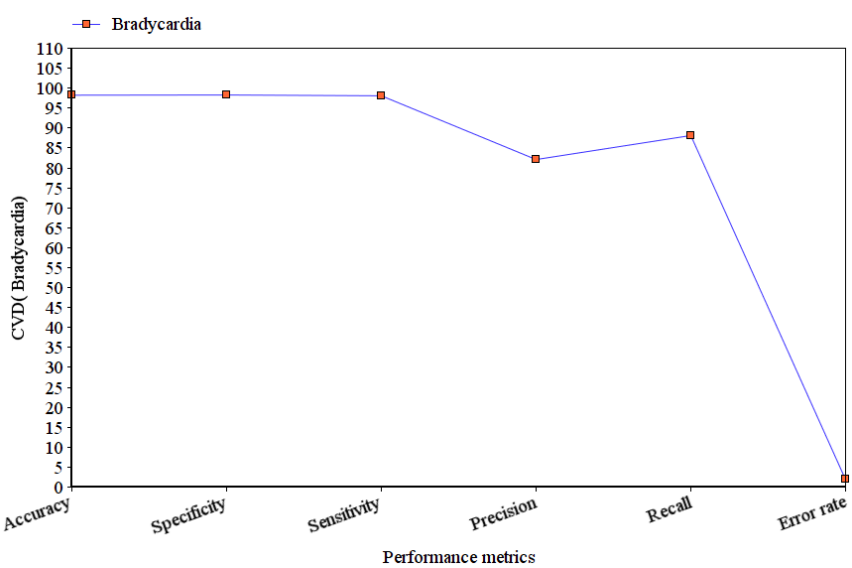

Figure 10. Performance results for predicting bradycardia
In this approach, the classification of Bradycardia provides $99.23 \%$ accuracy, $98.28 \%$ specificity, $98.08 \%$ sensitivity, $82.1 \%$ precision rate, $88.12 \%$ recall and $1.88 \%$ lower error rate that results are declared in Table 2. Performance analysis of model has been represented using ROC curve in Figure 11.

Table 2. Performance results for bradycardia

\begin{tabular}{cc}
\hline Performance Metrics & Percentage \\
\hline Accuracy & 98.23 \\
Specificity & 98.28 \\
Sensitivity & 98.08 \\
Precision & 82.1 \\
Recall & 88.12 \\
Error rate & 1.88 \\
\hline
\end{tabular}

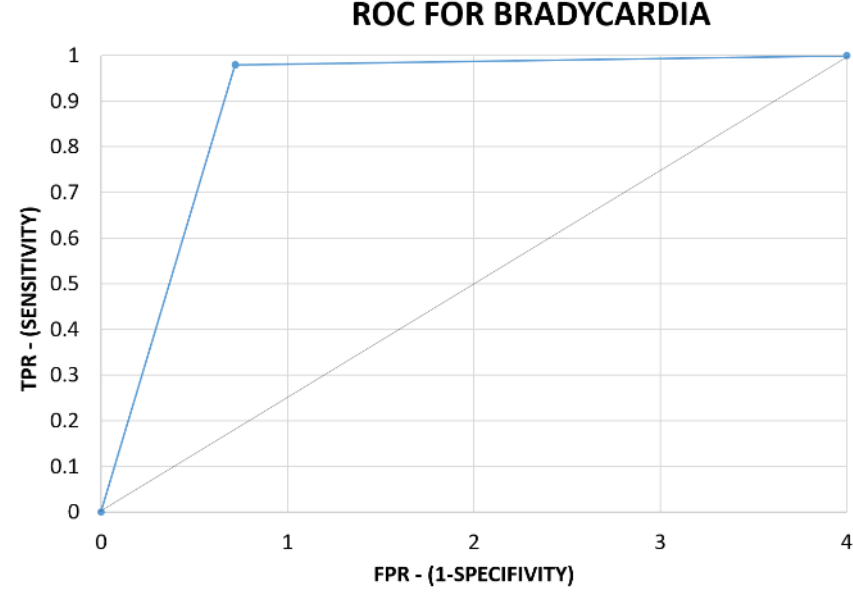

Figure 11. ROC for bradycardia

Angina is chest pain or discomfort caused when a heart muscle doesn't get enough oxygen-rich blood that may create pressure in the chest. The discomfort also can occur in shoulders, arms, neck, jaw, or back. Prediction results of Angina are mentioned in Figure 12.

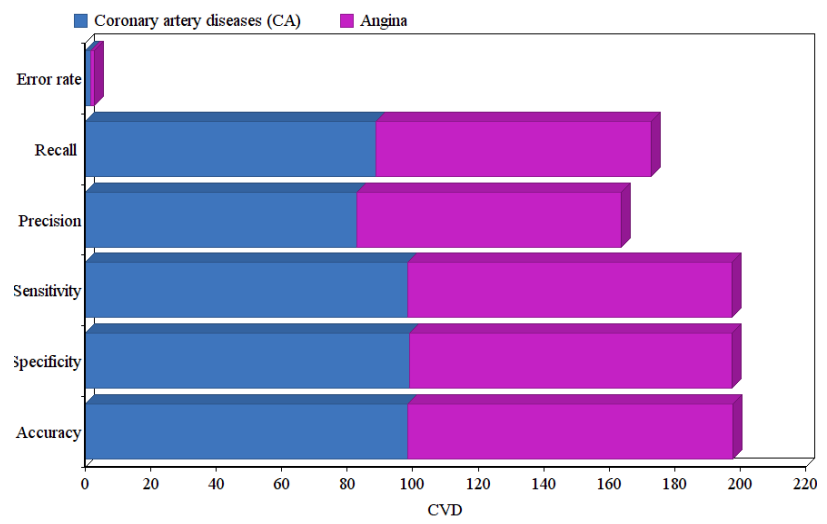

Figure 12. Performance results for predicting coronary artery diseases and angina

In proposed approach, the classification of Angina provides $99.1 \%$ accuracy, $98.58 \%$ specificity, $98.98 \%$ sensitivity, $80.8 \%$ precision rate, $83.98 \%$ recall and $0.9 \%$ lower error rate that results are detailed in Table 3. Performance analysis of model has been represented using ROC curve in Figure 13. 
Coronary artery disease is the narrowing or blockage of the coronary arteries, usually caused by atherosclerosis. Atherosclerosis is the buildup of cholesterol and fatty deposits on the inner walls of the arteries which are predicted and classified using CFuNM-HACABO approach and the performance measures are shown in Figure 12 and the values are mentioned in Table 4. Performance analysis of model has been represented using ROC curve in Figure 14.

Table 3. Performance results for angina

\begin{tabular}{cc}
\hline Performance Metrics & Percentage \\
\hline Accuracy & 99.1 \\
Specificity & 98.58 \\
Sensitivity & 98.98 \\
Precision & 80.8 \\
Recall & 83.98 \\
Error rate & 0.9 \\
\hline
\end{tabular}

Table 4. Performance results for coronary artery diseases

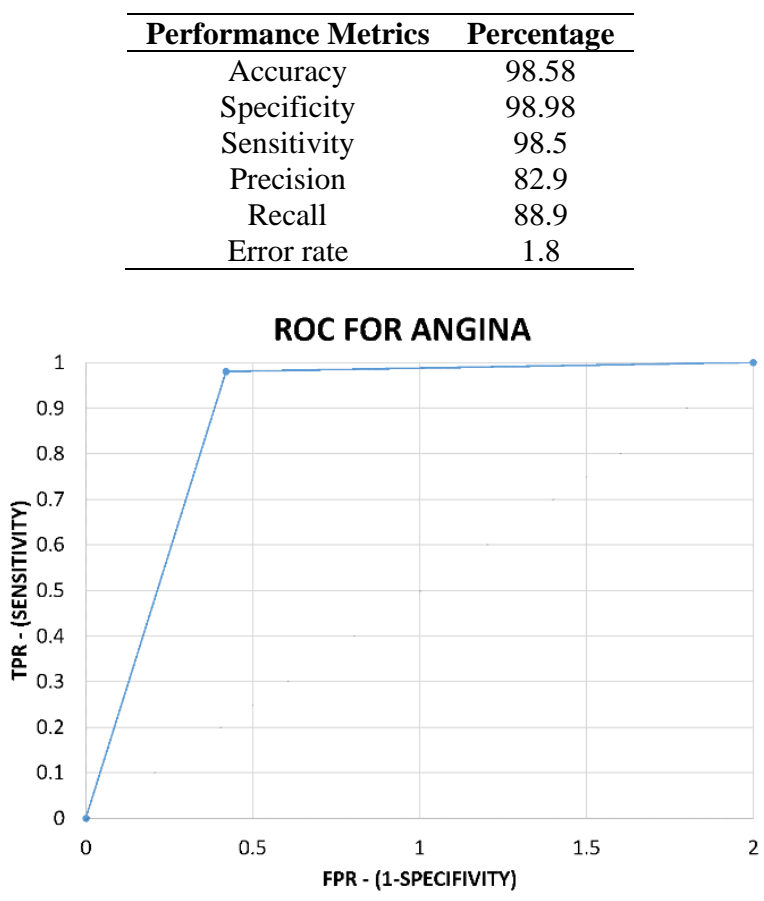

Figure 13. ROC for angina

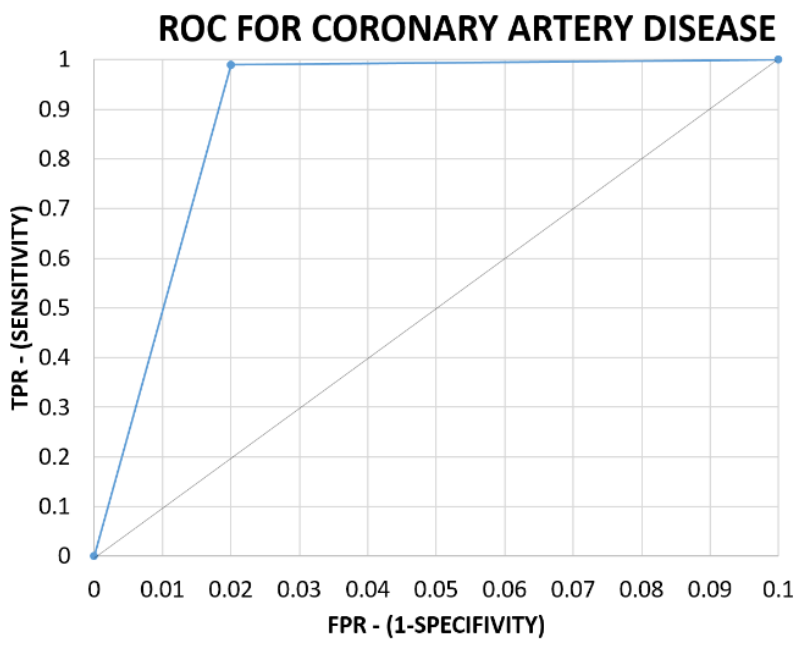

Figure 14. ROC for coronary artery disease
The proposed approach gives better accuracy and better precisions which is used to predict the various cardiovascular diseases. The proposed mechanism achieves accuracy as $99.39 \%$, sensitivity as $98.1 \%$ and specificity is calculated as $95.89 \%$. To compare the efficiency of proposed strategy with existing work ROC has been generated and represented in Figure 15, some of the techniques are obtained such as Machine Learning (ML) [24] and Cox regression analysis [25]

When performing the objective feature ranking the ML algorithms include the possibility to find new insights in real data. Compared to other methods such as machine learning and Cox regression analysis, the proposed method provides better accuracy, sensitivity, and specificity for predicting diseases that are shown in Figure 16.

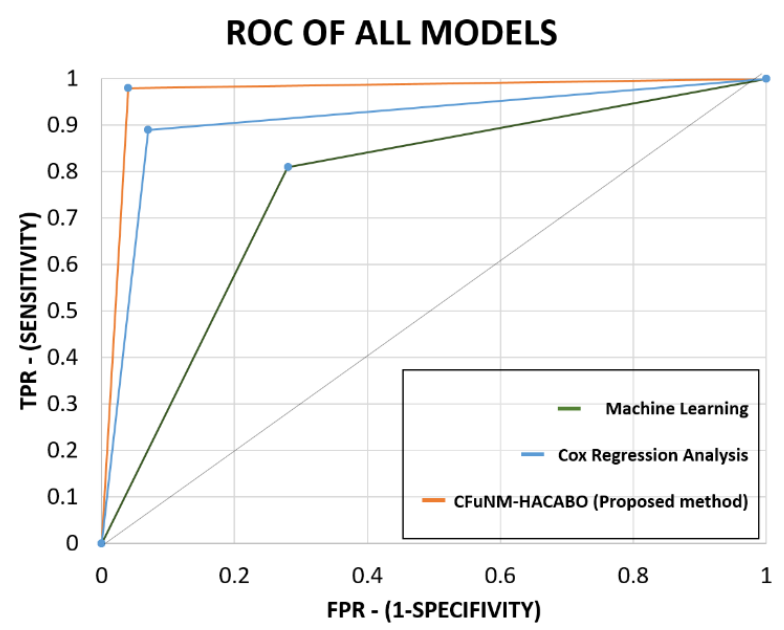

Figure 15. ROC - Performance of models

Table 5. Comparison for various segmentation techniques

\begin{tabular}{cccc}
\hline Techniques & Accuracy & Sensitivity & Specificity \\
\hline Machine Learning & 98 & 81 & 72 \\
Cox Regression & 98.28 & 88.8 & 92.8 \\
$\begin{array}{c}\text { Analysis } \\
\text { CFuNM-HACABO } \\
\text { (Proposed method) }\end{array}$ & 99.39 & 98.1 & 95.89 \\
\hline
\end{tabular}

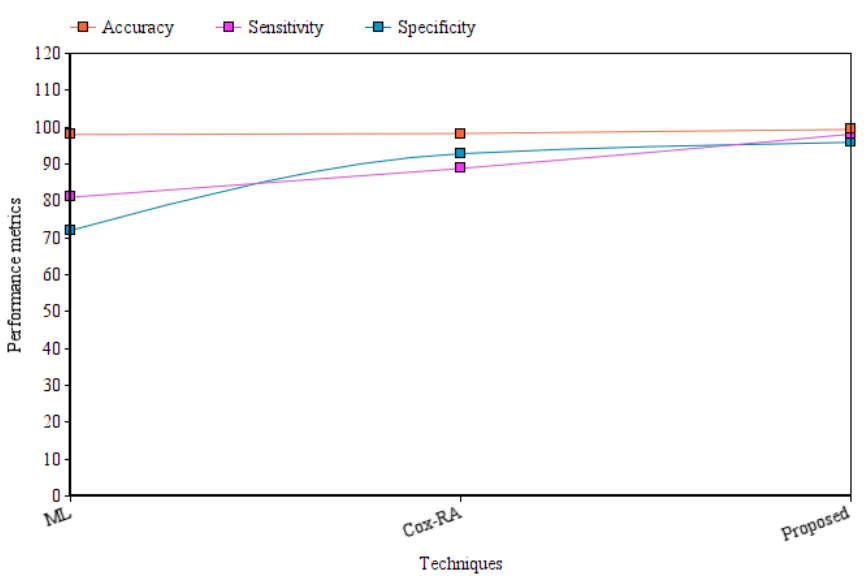

Figure 16. Comparison with existing methods

The evaluation of machine learning, Cox regression analysis method and our proposed technique explains in Table 5. Machine learning method is an amalgamation of experimental data and imaging measures used to improve prediction of analytical outcomes and shows patient risk 
prediction. The sensitivity values of existing approaches are $81 \%, 88.8 \%$ and the proposed method achieves high sensitivity as $98.1 \%$. The proposed approach also gives better specificity $95.89 \%$ value compared to other methods.

\section{CONCLUSION AND FUTURE SCOPE}

Findings in this research mainly focused on the study of MRI image towards the classification of cardiovascular diseases. In this proposed research classification is done using Cat Fuzzy Neural Model (CFNm) and hybrid optimization algorithm. HAC-ABO is used to evaluate better classification to predict the disease with $99.3 \%$ Accuracy with an error rate of $0.18 \%$. Moreover, the comparative result proved the efficiency of the proposed research. As meta heuristic optimization algorithms and hybrid optimization algorithms are implemented in this area of research in order to achieve more accurate results. Hence in our future endeavours we wish to work on more such methods along with feature selection mechanisms to develop hybrid method towards achieving more accurate prediction results on different image datasets.

\section{ACKNOWLEDGMENT}

I sincerely thank Dr. Debnath Bhattacharyya for his valuable guidance and support throughout this research work.

\section{REFERENCES}

[1] Chen, C., Qin, C., Qiu, H., Tarroni, G., Duan, J., Bai, W., Rueckert, D. (2020). deep learning for cardiac image segmentation: A review. Front Cardiovasc Med., 7: 25. https://doi.org/10.3389/fcvm.2020.00025

[2] Najafabadi, M.M., Villanustre, F., Khoshgoftaar, T.M., Seliya, N., Wald, R., Muharemagic, E. (2015). Deep learning applications and challenges in big data analytics. Journal of Big Data, 2: 1-21. https://doi.org/10.1186/s40537-014-0007-7

[3] http://en.wikipedia.org/wiki/Hann function, accessed on 13 June, 2020.

[4] Lyra, M., Ploussi, A. (2011). Filtering in SPECT image reconstruction. International Journal of Biomedical Imaging, 2011: 1-14. https://doi.org/10.1155/2011/693795

[5] Lehmann, T.M., Gonner, C., Spitzer, K. (1999). Survey: Interpolation methods in medical image processing. IEEE Transactions on Medical Imaging, 18(11): 1089 1085. https://doi.org/10.1109/42.816070

[6] Maier, A., Syben, C., Lasser, T., Riess, C. (2019). A gentle introduction to deep learning in medical image processing. Zeitschrift für Medizinische Physik, 29(2): 88-101. https://doi.org/10.1016/j.zemedi.2018.12.003

[7] Abdallah, Y.M.Y., Alqahtani, T. (2019). Research in medical imaging using image processing techniques. Medical Imaging-Principles and Applications. IntechOpen. https://doi.org/10.5772/intechopen.84360

[8] Ting, T.O., Yang, X.S., Cheng, S., Huang, K. (2014). Hybrid metaheuristic algorithms: Past, present, and future. In: Yang, X.S. (eds) Recent Advances in Swarm Intelligence and Evolutionary Computation. Studies in Computational Intelligence, 585: 71-83. https://doi.org/10.1007/978-3-319-13826-8 4

[9] Babu, K.R.R., Mathiyalagan, P., Sivanandam, S.N. (2014). Pareto based hybrid meta heuristic ABC - ACO approach for task scheduling in computational grids. International Journal of Hybrid Intelligent Systems, 11(4): 241-255. https://doi.org/10.3233/HIS-140197

[10] Moreno, S., Bonfante, M., Zurek, E., Juan, H.S. (2019). Study of medical image processing techniques applied to lung cancer. 2019 18th Iberian Conference on Information Systems and Technologies (CISTI) Coimbra, Portugal, pp.

$1-6$. https://doi.org/10.23919/CISTI.2019.8760888

[11] Maier-Hein, L., Eisenmann, M., Kopp-Schneider, A. (2019). Author correction: Why rankings of biomedical image analysis competitions should be interpreted with care. Nature Communications, 10: 588. https://doi.org/10.1038/s41467-019-08563-w

[12] Maier, A., Syben, C., Lasser, T., Riess, C. (2019). A gentle introduction to deep learning in medical image processing. Zeitschrift für Medizinische Physik, 29(2): 88-101. https://doi.org/10.1016/j.zemedi.2018.12.003

[13] Lenchik, L., Heacock, L., Weaver, A.A., Boutin, R.D., Cook, T.S., Itri, J., Filippi, C.G., Gullapalli, R.P., Lee, J., Zagurovskaya, M., Retson, T., Godwin, K., Nicholson, J., Narayana, P.A. (2019). Automated segmentation of tissues using CT and MRI: A systematic review. Academic Radiology, 26(12): 1695-1706. https://doi.org/10.1016/j.acra.2019.07.006

[14] Litjens, G., Ciompi, F., Wolterink, J.M., de Vos, B.D., Leiner, T., Teuwen, J., Išgum, I. (2019). State-of-the-art deep learning in cardiovascular image analysis. JACC: Cardiovascular Imaging, $12(8 \quad \mathrm{Pt} \quad 1)$ : 1589-1585. https://doi.org/10.1016/j.jcmg.2019.06.009

[15] Khanna, N.N., Jamthikar, A.D., Gupta, D., Piga, M., Saba, L., Carcassi, C., Giannopoulos, A.A., Nicolaides, A., Laird, J.R., Suri, H.S., Mavrogeni, S., Protogerou, A.D., Sfikakis, P., Kitas, G.D., Suri, J.S. (2019). Rheumatoid arthritis: atherosclerosis imaging and cardiovascular risk assessment using machine and deep learning-based tissue characterization. Current Atherosclerosis $\quad$ Reports, $21(2)$ : 7. https://doi.org/10.1007/s11883-019-0766-x

[16] Hasan, N.I., Bhattacharjee, A. (2019). Deep learning approach to cardiovascular disease classification employing modified ECG signal from empirical mode decomposition. Biomedical Signal Processing and Control, 52: 128-180. https://doi.org/10.1016/j.bspc.2019.04.005

[17] Luo, Y., Yang, B., Xu, L., Hao, L., Liu, J., Yao, Y., van de Vosse, F. (2018). Segmentation of the left ventricle in cardiac MRI using a hierarchical extreme learning machine model. International Journal of Machine Learning and Cybernetics, 9(10): 1881-1851. https://doi.org/10.1007/s13042-017-0678-4

[18] Podder, P., Khan, T.Z., Khan, M.H., Rahman, M.M. (2014). Comparative performance analysis of Hamming, Hanning and Blackman window. International Journal of Computer Applications, 96(18): 1-7. https://doi.org/10.5120/16891-6927

[19] Islam, M.Z., Islam, M.M., Asraf, A. (2020). A combined deep CNN-LSTM network for the detection of novel Coronavirus (COVID-19) using X-ray images. Informatics in Medicine Unlocked, 20: 1-20. https://doi.org/10.1101/2020.06.18.20134718 
[20] Ayon, S.I., Islam, M.M., Hossain, M.R. (2020): Coronary artery heart disease prediction: A comparative study of computational intelligence techniques. IETE Journal of

Research. https://doi.org/10.1080/03772063.2020.1713916

[21] Odili, J.B., Kahar, M.N.M., Anwar, S. (2015). African Buffalo optimization: A swarm-intelligence technique. Procedia Computer Science, 76: 443-448. https://doi.org/10.1016/j.procs.2015.12.291

[22] Andreopoulos, A., Tsotsos, J.K. (2008). Efficient and generalizable statistical models of shape and appearance for analysis of cardiac MRI. Medical Image Analysis, 12(3):

335-357. https://doi.org/10.1016/j.media.2007.12.003

[23] Abiyev, R., Arslan, M., Idoko, J.B., Sekeroglu, B., IIhan, A. (2020). Identification of epileptic EEG signals using
Convolutional Neural Networks. Applied Sciences, 10(12): 4089. https://doi.org/10.3390/app10124089

[24] Derchi, G., Dessì, C., Bina, P., Cappellini, M.D., Piga, A., Perrotta, S., Tartaglione, I., Giuditta, M., Longo, F., Origa, R., Quarta, A., Pinto, V., Forni, G.L., Webthal. (2019). Risk factors for heart disease in transfusiondependent thalassemia: Serum ferritin revisited. Internal and Emergency Medicine, 14(3): 385-380. https://doi.org/10.1007/s11739-018-1890-2

[25] Dey, D., Slomka, P.J., Leeson, P., Comaniciu, D., Shrestha, S., Sengupta, P.P., Marwick, T.H. (2019). Artificial intelligence in cardiovascular imaging: JACC state-of-the-art review. Journal of the American College of Cardiology, 73(11): 1318-1335. https://doi.org/10.1016/j.jacc.2018.12.054 